\title{
Mouse and Touchscreen Selection in the Upper and Lower Visual Fields
}

\author{
Barry A. Po, Brian D. Fisher, Kellogg S. Booth \\ Department of Computer Science, University of British Columbia \\ 201-2366 Main Mall, Vancouver, B.C. V6T 1Z4 \\ \{po, fisher, ksbooth\}@cs.ubc.ca
}

\begin{abstract}
Neuroanatomical evidence indicates the human eye's visual field can be functionally divided into two vertical hemifields, each specialized for specific functions. The upper visual field (UVF) is specialized to support perceptual tasks in the distance, while the lower visual field (LVF) is specialized to support visually-guided motor tasks, such as pointing. We present a user study comparing mouse- and touchscreen-based pointing for items presented in the UVF and LVF on an interactive display. Consistent with the neuroscience literature, we found that mouse and touchscreen pointing were faster and more accurate for items presented in the LVF when compared to pointing at identical targets presented in the UVF. Further analysis found previously unreported performance differences between the visual fields for touchscreen pointing that were not observed for mouse pointing. This indicates that a placement of interactive items favorable to the LVF yields superior user performance, especially for systems dependent on direct touch interactions.
\end{abstract}

Categories \& Subject Descriptors: H.1.2. [Models and Principles]: User/Machine Systems - Human factors; H.5.2. [Information Interfaces and Presentation (e.g., HCI)]: User Interfaces - Input devices and strategies (e.g., mouse, touchscreen)

General Terms: Design, Experimentation, Human Factors.

Keywords: Pointing, Visual Fields, Mice, Touchscreens, Fitts Law, Interactive Displays.

\section{INTRODUCTION}

Humans have evolved over a long period of time for environments considerably different from today's office, school, and home environments. With the advent of ubiquitous and immersive computing, there is a need to better understand the impact of evolved physiology on user performance. An ecologically-influenced approach to user

\footnotetext{
Permission to make digital or hard copies of all or part of this work for personal or classroom use is granted without fee provided that copies are not made or distributed for profit or commercial advantage and that copies bear this notice and the full citation on the first page. To copy otherwise, or republish, to post on servers or to redistribute to lists, requires prior specific permission and/or a fee.

CHI 2004, April 24-29, 2004, Vienna, Austria.

Copyright 2004 ACM 1-58113-702-8/04/0004...\$5.00
}

interface design takes into account the physiological limits of human ability. This can yield important insights for the design of future interactive systems.

One evolutionary aspect of the human visual system is how the anatomically-based functional division between the vertical receptive fields in the human eye emerged to differentially support activities in far (extrapersonal) space and near (peripersonal) space [7, 16, 19]. Visually-based activities in extrapersonal space, such as visual search and object recognition in the distance, are more efficient when conducted in the upper half of the perceived visual world the upper visual field (UVF). Likewise, visually-guided activities in peripersonal space, such as reaching, grasping, and pointing are more efficient in the lower half of the perceived visual world - the lower visual field (LVF).

To determine whether these functional differences influence user performance, we investigated mouse and touchscreen pointing on an interactive display, focusing on the neuroanatomical differences between the UVF and LVF. In a controlled user study, we found key performance differences in pointing-based selection of items, depending on whether items appeared in the UVF or LVF. Evidence of better performance in the LVF compared to performance in the UVF is consistent with differences reported in the neuroscience literature. We also observed differences between mouse and touchscreen interactions, which we interpret in the context of theories of the functional specialization of the visual fields. Based on these findings, we suggest design strategies for the placement of interactive elements in visual user interfaces and implications for future computing environments.

\section{BACKGROUND AND RELATED WORK}

Ecological theories postulate that major advances in the development of the primate visual system are responsible for the functional differences between the UVF and LVF $[16,19]$. These theories suggest that the visual environment of primates naturally segregated visual tasks into two distinct spaces. Far (or extrapersonal) space encompassed visual activities critical to survival that took place in the distance, such as the detection of colors specific to edible fruit and the recognition of facial expressions for nonverbal signaling and communication. In higher primates, an erect posture meant the eyes were elevated relative to the rest of the body, motivating a reliance on the upper half of what was seen for these activities. Thus, a functional ad- 
vantage for far space activities emerged in the upper visual field.

Near (or peripersonal) space encompassed visual activities critical to survival that took place within reaching distance, such as retrieving and ingesting fruits and other kinds of food. In higher primates, an erect posture meant the arms and hands were situated below eye level and were used primarily for manipulation, rather than for postural support. This motivated a reliance on the lower half of what was seen for these activities. Thus, a functional advantage for near space activities emerged in the lower visual field.

The neuroscience literature provides abundant evidence to support these ecological theories of functional division. Previc and Skrandies [16, 19] provide generous reviews of the experimental evidence indicating differences between the UVF and LVF. Their reviews cite numerous studies showing differences in reaction time performance, eye movements, visual thresholds, motion perception, visual attention, and visual evoked potentials. Other physiological evidence includes reports of non-uniform distributions of ganglion cells between the UVF and LVF, suggesting differences in information processing for each field $[5,6]$. A comparison of color persistence across the visual fields by Heider and Groner [9] also shows longer persistence in the UVF, which is consistent with the idea that the UVF is specialized for activities like object recognition.

Another study by Danckert and Goodale [7] cites evidence of direct connections between the LVF and brain areas responsible for visually-guided motor processing, consistent with a two-visual systems model of perception and action $[3,14,15]$. Their study shows superior direct pointing performance to targets presented in the LVF, consistent with the idea that the LVF is specialized for activities involving physical movement. Their two-visual systems interpretation is also consistent with a recent study by Po, Fisher, and Booth [15], which found superior pointing performance compared to voice input in a visually-biased target selection task on a large-screen display.

\section{Implications for $\mathrm{HCl}$}

The continual shift toward ubiquitous and immersive computing environments makes the segregation of far and near space a relevant design factor for HCI, and there are examples where this difference is important even in traditional desktop systems. By emphasizing the combined influence of attentional focus, eye position, item placement, and relative location on user performance, the differences between the UVF and LVF directly affect the usability of currentgeneration desktop user interfaces.

The implications of these performance differences easily extend to other kinds of user interfaces, such as vehicle interfaces and safety-critical systems. In these situations, efficient interactions and minimization of cognitive load are important factors in making these interfaces usable. When operating a vehicle, such as a car or an airplane, it is often infeasible or impossible to shift attention away from the primary task (i.e. driving) to complete a secondary task (i.e. changing dashboard settings). In designing these kinds of interfaces, understanding the differences between the UVF and LVF could be crucial in creating robust interfaces that minimize attentional shifts and accommodate multitasking in a cognitively-stressed environment. By arranging interfaces to support those activities most appropriate for the UVF and LVF, functional differences can be exploited.

\section{Mice and Touchscreens}

In desktop interaction, the mouse has played a critical role as a primary input device, and will likely continue to do so for some time. Nevertheless, touchscreen displays are quickly becoming an alternative medium for device interaction because they are easily adaptable to many different kinds of applications [18].

Both mice and touchscreens share certain characteristics that contribute to their popularity. First, they are highly precise and highly reliable technologies. Second, they are suitable for a wide range of interaction tasks, including target acquisition and tracing. Third, many of the interactive metaphors that apply to one technology also directly apply to the other (i.e. pointing).

In the context of pointing and target acquisition, Sears and Shneiderman made several direct comparisons between mice and touchscreens [17]. They compared performance speed, error rates, and user preference, providing evidence that properly used touchscreens yielded certain advantages in selecting targets as small as four pixels. They also found that touchscreen users were able to point at single pixel targets, suggesting that touchscreens can also be as precise as a mouse under ideal circumstances.

\section{Pointing and Fitts Law}

Both mice and touchscreens emphasize pointing interactions for target acquisition. Our user study evaluates the usability implications of the UVF and LVF by investigating their role in pointing performance. In both $\mathrm{HCI}$ and experimental psychology, a common technique to evaluate motor performance, such as pointing movement, is the application of Fitts Law [8, 13]. Derived from information theory, Fitts Law defines a log-linear relationship between movement time, target size, and target distance.

Fitts Law has been used extensively to model user performance in various interaction tasks including pointing and dragging, and in comparisons of various input devices [4, 12]. Numerous variants and extensions to Fitts Law have been used to better fit experimental data and to extend the basic model to other kinds of interaction tasks, such as trajectory-based interaction and bivariate pointing $[1,2]$.

In HCI, the most common formulation of Fitts Law states that movement time $(M T)$ is a function of target amplitude $(A)$ and width $(W)$ according to the following equation: 


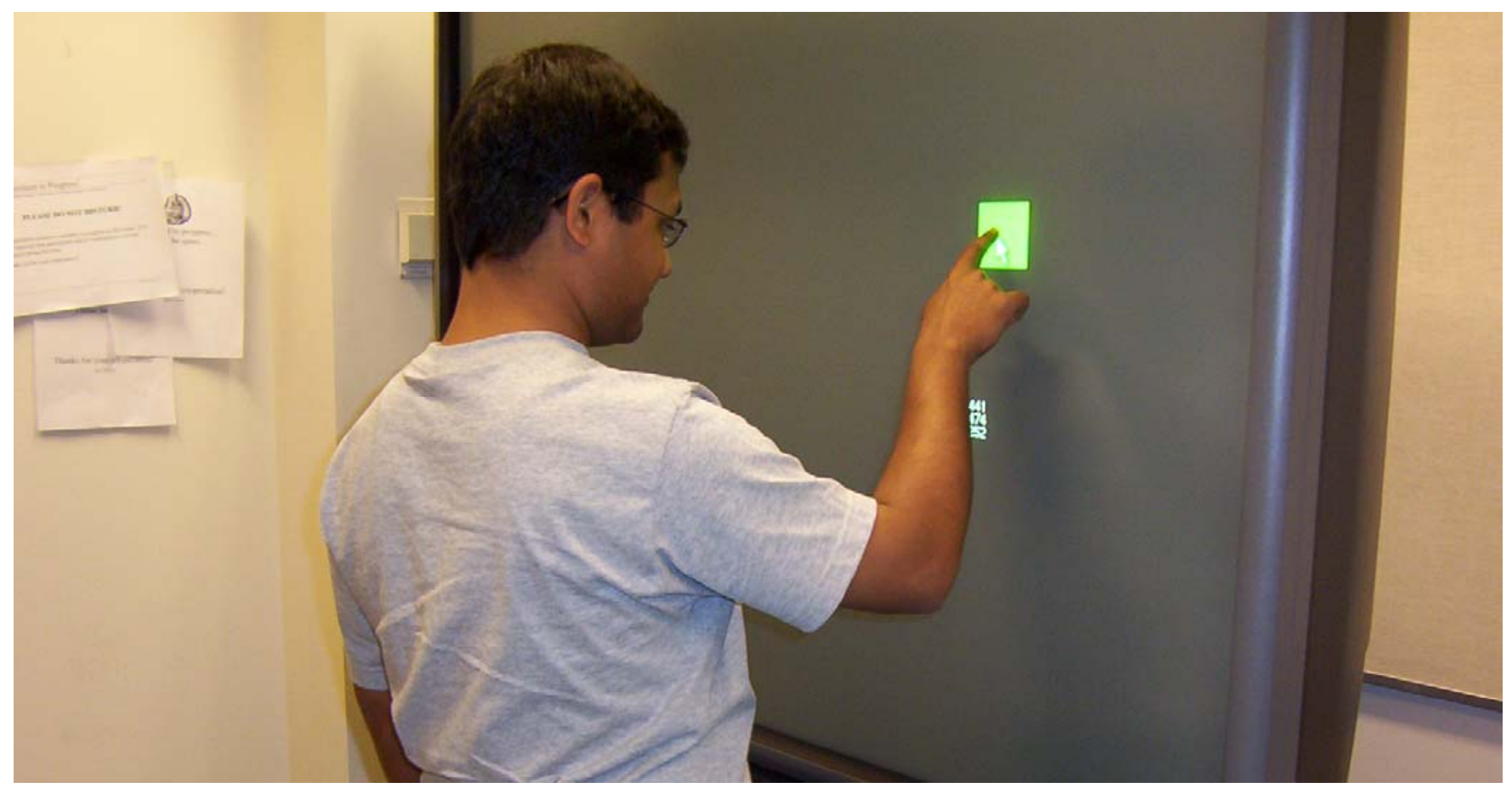

Figure 1. The SMART Board display used during the user study. Subjects stood upright during each block of trials. While fixating on a designated location on the screen, subjects either pointed directly at displayed items by touching them or indirectly by using an optical mouse (not shown). The designated location of fixation determined whether selected items appeared in the UVF or LVF.

$$
M T=a+b \log _{2}\left(\frac{A}{W}+1\right)
$$

The expression $\log _{2}\left(\frac{A}{W}+1\right)$ is commonly known as the index of difficulty (ID). The constants $a$ and $b$ are derived empirically and can be interpreted as the $y$-intercept and slope of a predictive linear regression equation. Thus, Fitts Law predicts a linear increase in movement time with a linear increase in the index of difficulty.

The above formulation of Fitts Law was originally proposed by MacKenzie [11] as an improvement to the original formulation by Fitts [8]. It is the standard formulation used to compare interaction devices and techniques in $\mathrm{HCI}$, and is the one used throughout this paper.

\section{HYPOTHESES}

Based on the evidence indicating functional differences between the UVF and LVF, user performance in an item selection task should differ depending on whether items to be selected are perceived in the UVF or LVF. Specifically, the physiological and experimental evidence to date suggest that:

1. Pointing performance should be markedly improved when the items to be selected are presented in the LVF, consistent with the claim that the LVF has an advantage for visually-guided motor responses [7].
2. There should be characteristic differences in the way that the LVF advantage exhibits itself between mice and touchscreen interactions, consistent with the claim that the LVF provides a distinct neural advantage for direct (touch) pointing, but not necessarily for indirect (mouse) pointing $[7,14]$.

In our user study, we tested these predictions by comparing empirically-derived performance modeled by Fitts Law. If pointing performance is better in the LVF, then there should be fundamental differences between the regression models generated between the UVF and LVF. Furthermore, there should be distinct differences when these models are compared between mouse and touchscreen pointing.

\section{METHODS}

To test our formal hypotheses, we developed a Fitts-like pointing task to compare mouse and touchscreen pointing performance between the two visual fields. Situating our study in an environment contextually-appropriate for HCI, we used a fully counterbalanced, within-subjects experimental design. Subjects were required to point at individual items of different widths and at different distances presented on a large-screen display. Each subject completed two blocks of pointing trials. One block consisted of touchscreen pointing trials and another block consisted of mouse pointing trials. These were counterbalanced such that half of the subjects completed mouse pointing before touchscreen pointing, while the other half completed touchscreen pointing before mouse pointing. 


\section{Subjects}

Eight subjects participated in the study. Five were male, and three were female. Their ages ranged from 19 to 40 years. All subjects were right-handed and had normal or corrected-to-normal vision.

\section{Apparatus}

Figure 1 depicts the display and experimental apparatus as it was arranged for the study. A pre-calibrated, touchsensitive SMART Board 3000i was used as the primary interactive display. The display was rear-projected and had an active LCD display area of approximately $136 \mathrm{~cm}$ by $102 \mathrm{~cm}$, running at a resolution of $1024 \times 768$ pixels. A connected PC workstation ran experimental software that presented trials and recorded subject performance data. Movement times were measured using a very highresolution timer with a sampling resolution of 3.6 microseconds. During the mouse pointing blocks, a standard Logitech optical Wheel Mouse was made available to subjects. While subjects were completing the experiment, a constant level of illumination was maintained.

Prior to each session, subjects were instructed to stand in front of the SMART Board while the experimenter adjusted the experiment software to ensure that items to be selected in trials would always appear at eye level. This calibration for individual height differences ensured that every subject saw the same rendered display, regardless of height.

\section{Procedure}

Each subject participated in a single session lasting approximately forty minutes. During a session, an experimenter was present at all times. In both mouse and touchscreen blocks, subjects stood upright before the SMART Board at a viewing distance of approximately 30 centimeters. Trials consisted of a single right-to-left pointing motion from a starting point to a single displayed item while fixating on a designated area of the screen whose vertical position was either above or below the pointing activity. These two fixation points allowed us to experimentally control whether display items appeared in the UVF or LVF.

Displayed items were single square targets appearing in one of four sizes: $8 \times 8,16 \times 16,32 \times 32$, or $64 \times 64$ pixels. At the subject viewing distance of about $30 \mathrm{~cm}$, these targets subtended approximately 1, 2, 4, and 8 degrees of visual angle, respectively. Targets appeared at one of four distances, or pointing amplitudes, from the starting position: $32,64,128$, or 256 pixels. At the subject viewing distance, these yielded approximately $4,8,16$, or 32 degrees of pointing movement, respectively. Because all pointing movements were made from right-to-left along the horizontal dimension, the pointing task was effectively limited to one movement dimension at all times. Each possible combination of target size, target distance and fixation point was repeated three times, yielding a total of $4 \times 4 \times 2 \times 3=96$ trials per block. Across mouse and touchscreen pointing blocks, subjects completed a total of 192 pointing trials.
All items were rendered against a black background. Individual trials within blocks were initiated by having subjects point and hold their aim at a $48 \times 48$ pixel starting square to their immediate right. A 5x3 array of randomly-generated numbers between 0 and 9 appeared 135 pixels, or about 20 degrees of visual angle, above or below eye-level. These arrays of numbers were rendered in a white, fixed $10 \times 10$ font. When subjects fixated upward, presented items appeared in the LVF. Conversely, when subjects fixated downward, items appeared in the UVF.

Subjects were instructed to point at the centre of displayed items and to emphasize speed and accuracy equally. They were also instructed to fixate on the designated fixation area at all times, even while pointing. After a period of 3 seconds, the $5 \times 3$ array of numbers was replaced by a single, randomly-generated number between 0 and 9 in the same font and style, and the displayed item to be selected appeared. Subjects verbally indicated the number to the experimenter while pointing at the displayed item. Subjects who incorrectly reported numbers or were observed to shift their gaze while pointing would have those trials invalidated, although this never happened in the pool of subjects that participated. The display was reset to the beginning of a new trial upon completion of the pointing movement and item selection.

The vertical fixation/response mechanism not only allowed us to experimentally control whether items appeared in the UVF or LVF, but also allowed us to do so without changing the physical mechanics of the pointing interaction. This mechanism also provided a suitable measure of certainty that subjects maintained presented items in the UVF or LVF at all times. If the subjects chose to "cheat" by fixating on the region of displayed item positions instead of the area of fixation, they would be unable to correctly report the final presented number in the fixation area while pointing because it would be very difficult to spatially individuate the number without re-fixating on it [10]. Subjects would be at a considerable disadvantage if they employed other fixation "strategies" such as indicating the number first and then re-fixating to point at the displayed item because this would slow them down considerably and would extend the duration of individual trials, thereby extending the length of the experiment. Subjects were highly competent in completing trials. Having subjects verbally indicate numbers while pointing did not seem to interfere with the primary pointing task.

\section{Mouse Condition}

In the block of mouse interaction trials, subjects were provided with a simple Logitech optical Wheel Mouse. The mouse was placed on a stable surface that was adjusted so that subjects of varying heights could use the mouse comfortably. Subjects pointed at onscreen items by aiming a rendered mouse cursor at items and left-clicking them. During trials, the mouse cursor did not move from the starting position until the final displayed item appeared. 


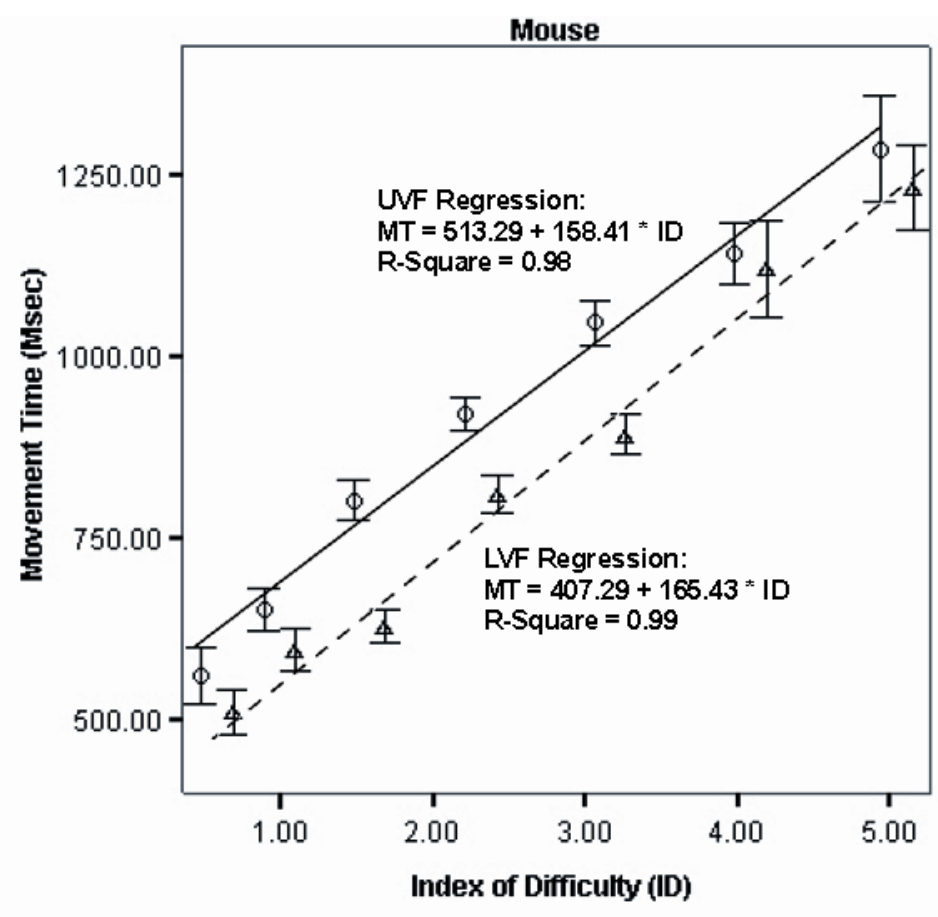

Error Bars show Mean $+1-1.0 \mathrm{SE}$
Touch

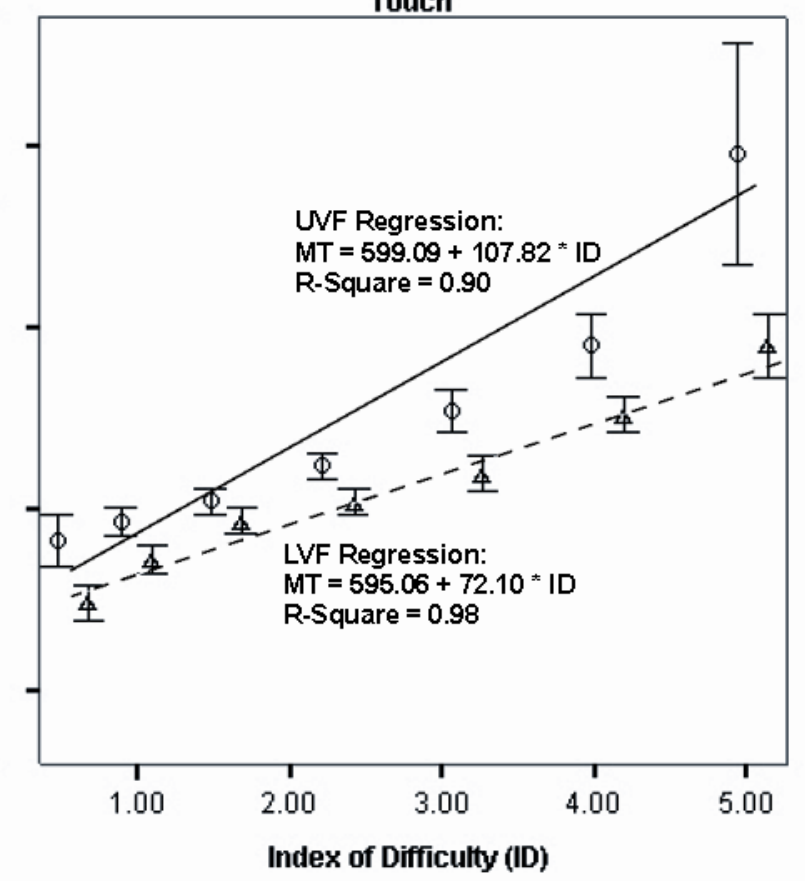

Figure 2. Linear regression plots for index of difficulty (ID) versus movement time (MT). The LVF has a distinct advantage in movement performance in all conditions, as shown by the more favorable LVF regressions. The linear fit generated for the UVF in touch pointing has a poorer fit compared to all other models. A combined linear and quadratic curve fits best for this condition.

\section{Touchscreen Condition}

In the block of touchscreen interaction trials, subjects directly pointed at displayed items by touching them with their index finger. At the start of a trial, subjects physically pointed and held their index finger down on the starting position until the final displayed item appeared. Subjects pointed at the displayed item by lifting their finger from the start position and tapping the item with the same finger.

\section{Training}

All subjects received a minimum of twenty practice trials prior to each block of experimental trials. Practice trials consisted of trials presented in the same fashion as the experimental trials, with randomized item sizes, distances, and fixation points. Practice trials were presented until both subject and experimenter were satisfied with the subject's ability to complete the task properly.

\section{RESULTS}

Least-squares linear regressions were used to analyze movement time against calculated indices of difficulty across aggregate pointing data from all subjects. Separate linear regressions were performed for each combination of visual field (UVF or LVF) and interaction style (mouse or touchscreen). Trend analyses and curve estimation regression analyses were performed to find lines of best fit for each of visual field and interaction style. Pointing accuracy was quantitatively assessed using a radial error metric that calculated the square root of the sum of squared horizontal and vertical displacements from the centre of each presented item (i.e. Error $=\sqrt{x^{2}+y^{2}}$ ). This metric was applied to subjects and accuracy differences were analyzed using two-way ANOVAs with repeated measures against visual field and index of difficulty for each interaction style.

\section{Movement Time Performance}

Figure 2 presents the linear regression plots for the calculated indices of difficulty (ID) versus movement time (MT). When movement time performance is compared across the visual fields, it is clear there are performance differences in favor of the LVF. In the mouse and touchscreen conditions, the regression equations yield smaller $y$-intercept values, and in the case of touchscreen pointing, yield a noticeably shallower linear slope. Thus, movement times to displayed items were consistently faster when they were presented in the LVF. 


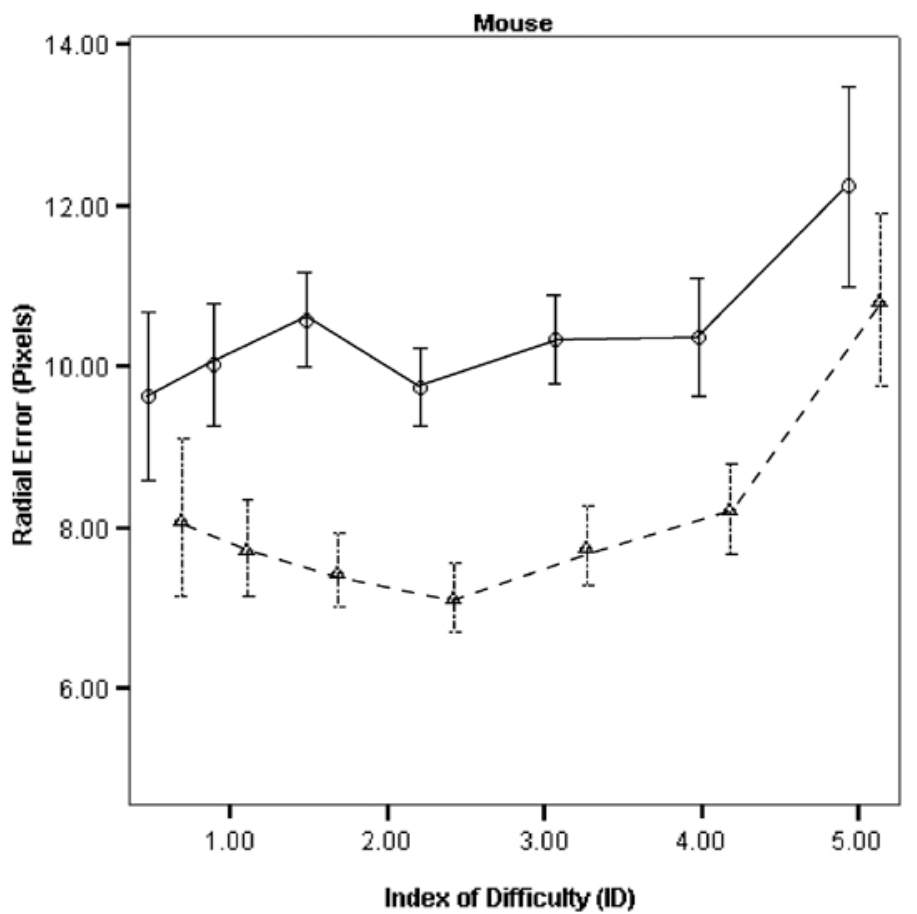

Index of Difficulty (ID)

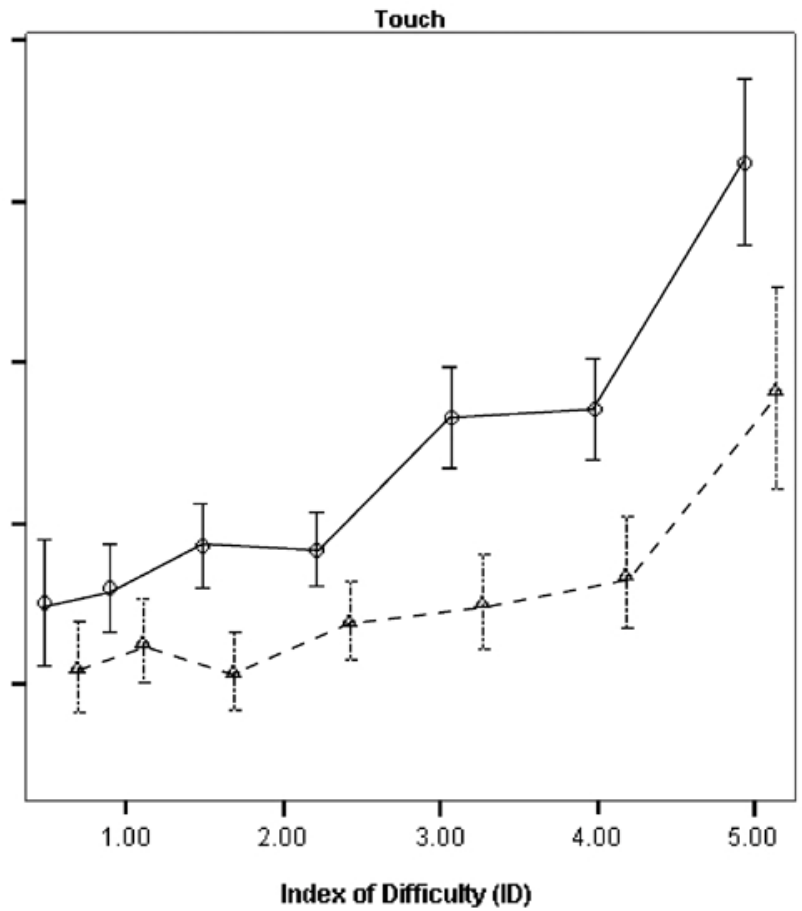

Error Bars show Mean $+1-1.0 \mathrm{SE}$
Fixation Point (UVFI LVF) O- UVF
$-\frac{\mathrm{A}}{\mathrm{x}}-\mathrm{LVF}$

Figure 3. Line graph of index of difficulty plotted against radial error (pointing accuracy in pixels). The LVF shows consistently better accuracy when compared against pointing to displayed items in the UVF.

For mouse interaction, the regressions for both the UVF and LVF are consistent with the performance patterns predicted by Fitts Law, with very high $r^{2}$ values ( 0.98 and 0.99 respectively), which indicate a very high degree of correlation between the predicted regression and sampled data. For touchscreen interaction, the regression for the LVF is also consistent with Fitts Law with an equally high $r^{2}$ value (0.98). However, the UVF regression exhibits an $r^{2}$ value that is comparatively smaller $(0.90)$. Informal analysis of the regression plot suggests a non-linear equation might provide a better fit to the sampled data.

The formal series of trend analyses conducted across visual fields and interaction styles provide support for this observation. In the case of the UVF and touchscreen pointing, a trend analysis for polynomial contrasts indicates that a combined linear and quadratic fit would account for a greater proportion of the variance (contrast $p<0.001$; deviation $p<0.001$ ) than a linear fit alone (linear $\eta^{2}=0.90$; linear and quadratic $\eta^{2}=0.98$ ). Other higher-order contrasts were not significant in this condition and only linear contrasts were statistically significant for all other conditions. Curve estimation regressions also concurred with the trend analyses, indicating that a quadratic equation would fit better than a linear regression equation (quadratic equation: $\left.\mathrm{MT}=737.99+26.06 * \mathrm{ID}^{2}-36.83 * \mathrm{ID} ; r^{2}=0.98\right)$. Thus, the data indicates that Fitts Law was obeyed with less fidelity under touchscreen interaction in the UVF, especially at higher indices of difficulty.

\section{Pointing Accuracy}

Figure 3 is a line graph plotting index of difficulty (ID) against pointing accuracy, as measured by the previously described radial error metric. Similar to movement time, there are observable differences in accuracy across the visual fields, with consistently better accuracy in the LVF. A two-way ANOVA with repeated measures against visual field and index of difficulty for each interaction style indicates statistically significant main effects for visual field $[\mathrm{F}(1,21)=15.294, p<0.001$ for mouse; $\mathrm{F}(1,21)=10.204$, $p=0.004$ for touchscreen] and index of difficulty $[\mathrm{F}(6$, $126)=2.667, p=0.018$ for mouse; $\mathrm{F}(6,126)=4.577, p<$ 0.001 for touchscreen]. There were no significant two-way interactions between visual field and index of difficulty for either mouse or touchscreen interaction styles. As might be expected, these results meant displayed items with higher indices of difficulty were more difficult to acquire accurately. The independent main effect of visual field also indicated that perceived location relative to fixation was important: pointing accuracy improved by simply changing the visual field in which displayed items appeared.

\section{Other Effects}

Group means between the visual fields and interaction 
styles were analyzed with paired-sample $t$-tests to characterize overall performance differences. All tests between the visual fields were highly significant, indicating faster movement times and greater accuracy when subjects pointed at displayed items in the LVF (mouse, movement time: $t(7)=3.488, p=0.010$; mouse, radial error: $t(7)=$ $2.500, p=0.041$; touch, movement time: $t(7)=4.259, p=$ 0.004 ; touch, radial error: $t(7)=3.641, p=0.008$ ). Mouse pointing exhibited a mean movement time difference of $106 \mathrm{~ms}$ and touchscreen pointing exhibited a mean difference of about $143 \mathrm{~ms}$ between the UVF and LVF. Mouse and touchscreen pointing exhibited mean pointing accuracy differences of approximately 3 pixels and 2 pixels radial error respectively across the visual fields. Moreover, these time and accuracy differences grew considerably with increases in the index of difficulty.

In relative terms, pointing to displayed items in the LVF was $11.5 \%$ faster and approximately $29 \%$ more accurate than pointing to the same items in the UVF when using a mouse. Likewise, pointing to items in the LVF was $16 \%$ faster and about $24 \%$ more accurate than pointing to the same items in the UVF when using a touchscreen. From a practical standpoint, this suggests the differences between the UVF and LVF are quite important, especially when considering the design of visual interfaces where response time and low user error rates are of high importance.

Alternative analyses were performed to provide greater confidence that the observed differences were not simply present by chance. A series of linear regressions were performed to analyze the individual differences across the visual fields and interaction styles for each individual subject. Thus, eight regressions were generated - one for each subject. This kind of analysis is consistent with experimental psychophysics and alternative methods of statistical inference, which emphasize the importance of characterizing performance at the level of the individual [20].

While the regression coefficients differed from subject to subject, the linear regressions from all eight subjects individually exhibited regression equations in favor of the LVF in a manner similar to the aggregate data. This was consistently true across mouse and touchscreen interaction. Three of the eight subjects also exhibited a non-linear trend for touchscreen movement time in the UVF, suggesting the non-linear trend exhibited in the aggregate analysis may not necessarily be the result of statistical outliers, but may in fact be a symptom of characteristic individual differences between different subjects.

\section{DISCUSSION AND FUTURE WORK}

The results clearly support our hypothesis that displayed items are more efficiently selected when they are perceived in the LVF. The non-linear fit observed in the UVF for touchscreen interaction also lends evidence to our hypothesis that there may be different neural mechanisms at work when either directly pointing or indirectly pointing with a mouse. This could indicate that the way users implicitly process perceived information differs depending on the interaction style being used, meaning the perceived location of interactive elements becomes even more important as systems move toward more direct styles of interaction.

In the context of the functional dichotomy between the UVF and LVF, this difference between mouse and touchscreen performance could ultimately suggest the presence of an important theoretical limit for direct visually-guided motor performance, where an increase in cognitive activity is required for interaction in the extreme visual periphery. However, with the limited number of subjects and the design limitations of the current study, it is difficult to be certain. Future work in this area is required.

Our present results suggest a number of strategies and future implications for user interface design:

1. Ideally, the most frequently selected and most important interactive elements should be located in the lower half of a display. Items located in the lower half of a display are more likely to be initially perceived in the LVF. Following this simple placement strategy optimizes user performance by facilitating use of the LVF for interaction. This especially applies to interface elements that demand greater attentional resolution, where the LVF is functionally specialized.

2. Ideally, physically direct interactions, such as touchscreen pointing, should be preferred over less direct interactions, such as mouse pointing. Our study results are consistent with the presence of a neural advantage for physically direct interactions, which is especially evident when items are presented to the LVF. Following this guideline optimizes user performance by facilitating use of this advantage. This is especially important for situations where users may need to select individual items rapidly and repeatedly.

3. Ideally, adopt a strategy of organizing an interface for perception in the UVF and interaction in the LVF. Taking advantage of the functional specializations of the UVF and LVF means designers can optimize user performance by streamlining the processing of visual information. This is especially important when an interface is visually complex and demands a great deal of users' cognitive and attentional resources.

As physically direct interaction becomes more common, there will be a stronger relationship between visual perceptual processing at the physiological level and users' corresponding ability to efficiently interact with systems. Developers will need to learn how to design interactive systems based on the user performance limitations dictated by human physiology in order to achieve optimal usability. These strategies demonstrate how underlying physiological processes can be exploited to reduce cognitive load and incrementally improve user performance. While the performance gains derived from simply placing interactive items to favor the LVF might seem negligible in desktop 
GUI scenarios, they may be compelling where it is not always feasible to directly fixate on interactive elements (i.e. driving) or where there is low tolerance for user errors.

In the future, a more thorough understanding of the functional specializations of the UVF/LVF could lead to some very intriguing applications. For example, future advances in eye-tracking technology may benefit from an understanding of these UVF/LVF specializations. Adaptive eye tracking could be used to optimize use of system resources by differentially presenting higher-resolution imagery to the LVF and lower-resolution imagery to the UVF in a perceptually-based rendering scenario. Similarly, characterizing the UVF/LVF for predictive eye tracking could be used in conjunction with knowledge of gaze direction to dynamically optimize the layout of an interface for specific activities. Designers may also learn how to optimize the layout of a user interface to permit the cognitive "pipelining" of interactions. Such interfaces would be designed to facilitate the subliminal execution of an interaction concurrent with user planning of subsequent interactions.

\section{CONCLUSION}

We presented a user study that compared mouse and touchscreen performance in an item selection task across the upper and lower visual fields. Consistent with the neuroscience literature, our results indicate faster and more accurate item selection when items are presented in the LVF. These performance differences have definite implications for user interface design, especially for time-and safety-critical systems and future ubiquitous interfaces, where there is a greater degree of separation between near and far visual space, and systems may be largely dependent on direct touch-style interactions.

\section{ACKNOWLEDGEMENTS}

Financial support for this work was provided by the research and strategic grant programs of the Natural Sciences and Engineering Research Council of Canada (NSERC).

\section{REFERENCES}

[1] Accot, J., and Zhai, S. Beyond Fitts' law: Models for trajectory-based HCI tasks. Proc. ACM CHI 1997, 295-302.

[2] Accot, J., and Zhai, S. Refining Fitts' law models for bivariate pointing. Proc. ACM CHI 2003, 193-200.

[3] Bridgeman, B., Peery, S., and Anand, S. Interaction of cognitive and sensorimotor maps of visual space. Perception and Psychophysics 59(3), (1997), 456-469.

[4] Card, S. K., English, W. K., and Burr, B. J. Evaluation of mouse, rate-controlled isometric joystick, step keys, and text keys for text selection on a CRT. Ergonomics 21 (1978), 601-613.

[5] Curcio, C. A., and Allen, K. A. Topography of ganglion cells in human retina. J. Comp. Neurol. 300(1), (1990), 5-25.
[6] Curcio, C. A., Sloan, K. R., Packer, O., Hendrickson, A. E., and Kalina, R. E. Distribution of cones in human and monkey retina: individual variability and radial asymmetry. Science 236(4801), (1987), 579-581.

[7] Danckert, J., and Goodale, M. A. Superior performance for visually guided pointing in the lower visual field. Exp. Brain Res. 137 (2001), 303-308.

[8] Fitts, P. M. The information capacity of the human motor system in controlling amplitude of movement. Journal of Exp. Psychology 47 (1954), 381-391.

[9] Heider, B., and Groner, R. Vertical visual-field differences in schematic persistence for colour information. Proc. European Conference on Visual Perception 1997.

[10] Intriligator, J. and Cavanagh, P. The spatial resolution of visual attention. Cognitive Psychology 43 (2001), 171-216.

[11] MacKenzie, I. S. A note on the information-theoretic basis for Fitts' law. Journal of Motor Behavior 21 (1989), 323-330.

[12] MacKenzie, I. S., Sellen, A., and Buxton, W. A comparison of input devices in elemental pointing and dragging tasks. Proc. ACM CHI 1991, 161-166.

[13] MacKenzie, I. S. Fitts' Law as a performance model in human-computer interaction. Human-Computer Interaction 7(1), (1992), 91-139.

[14] Milner, A. D., and Goodale, M. A. The Visual Brain in Action. Oxford Psychology Series 27. Oxford University Press, New York, 1995.

[15] Po, B. A., Fisher, B. D., and Booth, K. S. Pointing and visual feedback for spatial interaction in large-screen display environments. Proc. of the $3^{\text {rd }}$ International Symposium on Smart Graphics 2003, 22-38.

[16] Previc, F. H. Functional specialization in the lower and upper visual fields in humans: Its ecological origins and neurophysiological implications. Behavioral and Brain Sciences 13 (1990), 519-575.

[17] Sears, A., and Shneiderman, B. High precision touchscreens: Design strategies and comparisons with a mouse. International Journal of Man-Machine Studies 43(4), (1991), 593-613.

[18] Shneiderman, B. Touch screens now offer compelling uses. IEEE Software 8(2), 1991, 93-94, 107.

[19] Skrandies, W. The upper and lower visual field of man: Electrophysiological and functional differences. Progress in Sensory Physiology 8. Springer-Verlag, Germany, (1987), 162-189.

[20] Vicente, K. J., and Torenvliet, G. L. The Earth is spherical $(p<0.05)$ : Alternative methods of statistical inference. Theor. Issues in Ergon. Sci 1(3), (2000), 248-271. 